Modeling, Identification and Control, Vol. 40, No. 4, 2019, pp. 189-198, ISSN 1890-1328

\title{
Payload estimation using forcemyography sensors for control of upper-body exoskeleton in load carrying assistance
}

\author{
Muhammad R. U. Islam ${ }^{1}$ Shaoping Bai ${ }^{1}$ \\ ${ }^{1}$ Department of Materials and Production, Aalborg University, Aalborg, Denmark. E-mail: (mraza,shb)@mp.aau.dk
}

\begin{abstract}
In robotic assistive devices, the determination of required assistance is vital for proper functioning of assistive control. This paper presents a novel solution to measure conveniently and accurately carried payload in order to estimate the required assistance level. The payload is estimated using upper arm forcemyography (FMG) through a sensor band made of force sensitive resistors. The sensor band is worn on the upper arm and is able to measure the change of normal force applied due to muscle contraction. The readings of the sensor band are processed using support vector machine (SVM) regression technique to estimate the payload. The developed method was tested on human subjects, carrying a payload. Experiments were further conducted on an upper-body exoskeleton to provide the required assistance. The results show that the developed method is able to estimate the load carrying status, which can be used in exoskeleton control to provide effectively physical assistance needed.
\end{abstract}

Keywords: Forcemyography, payload estimation, assistive exoskeleton, physical human-robot interaction.

\section{Introduction}

With the advancement in robot technology, exoskeletons are being developed for medical, industrial and service applications. Based on the applications, exoskeletons are categorized in three types i.e. rehabilitation, assistance and power augmentation exoskeletons Fan and Yin (2013); Hsieh et al. (2017); Cui et al. (2016); Keller et al. (2016); Huang et al. (2015); Castro et al. (2019); Christensen and Bai (2018); Gunasekara et al. (2012); Zhou et al. (2015). Rehabilitation and power augmentation exoskeletons are mainly focused on serving humans to regain their mobility and helping the users with extra power to enhance their capability, respectively Bai et al. (2018). In this work, our interest is to use exoskeletons to assist users, which can be either factory workers, elderly or person weak muscle strength, in load carrying tasks.

For physical assistance exoskeletons, the determina- tion of required assistance level is one of primary concerns. In load carrying tasks, one method to determine required assistance level is by knowing the payload value and joint configuration. In existing upper body exoskeleton systems, payload information is acquired by integrating force sensors at the end-effector, where the weight is hanged on to the exoskeleton and not carried by the human Rosen et al. (2001); Lee et al. (2014). This method is useful in specific applications, particularly heavy-load carrying tasks. The implementation of this method for daily routine activities or factory tasks is not feasible, where the user carries objects of different attributes. To overcome this challenge, Electromyography (EMG) based estimation methods are used instead to determine joint torques and provide assistance through exoskeletons McDonald et al. (2017); Mangukiya et al. (2017); Leonardis et al. (2015); Abdallah et al. (2017); Mghames et al. (2017); Tang et al. (2014); Rahman et al. (2015); Li et al. (2013); Kiguchi 
and Hayashi (2012). In these methods the assistance to each joint is provided by analyzing the muscle activities of its prime mover muscle group. In upper body multi DOF exoskeleton, this approach brings computational complexity, as large number of EMG electrodes need to be processed. Moreover, for daily usage placement of EMG electrodes to right place, proper skin preparation, low $\mathrm{S} / \mathrm{N}$ ratio and convenience are other challenges to be addressed.

In this work, we proposed FMG based method to estimate the payload level. This method requires no load cell to be attached at the end-effector and only requires an Force Sensitive Resistor (FSR) sensor band to perceive upper arm muscles activity and joint configuration in estimating payload level. Compared with other methods like EMG sensors, the new method requires simple electronics. $\mathrm{S} / \mathrm{N}$ ratio is also better and is not affected by skin condition.

In literature, there are some reported works on using FMG to detect upper body and lower body movements Cho et al. (2016); Kadkhodayan et al. (2016); Xiao and Menon (2017); Sadarangani and Menon (2017); Islam and Bai (2017); Jiang et al. (2016); Xiao et al. (2014); Islam et al. (2018). However, this approach has not been used yet for payload estimation to control upperbody exoskeleton in load carrying tasks. In this work, we developed an FSR sensor band, which measures the normal force applied by the muscles as they contract. The required assistance level is then estimated from the sensor readings, processed by machine learning, in terms of carried payload. The new method provides convenient and accurate estimates of payload carried by a person.

This paper is organized as follow: Design and implementation of the sensor band is described in Section 2. Section 3 presents the algorithm design of payload estimation. Experimental setup for sensor testing and its results are described in Section 4. In Section 5, we include briefly exoskeleton control with the developed sensor band to demonstrate the application. Discussion on the developed method is presented in Section 6 and the work is concluded in Section 7.

\section{Sensor band design}

Figure 1 shows an FSR sensor band developed. When the sensor band is worn on an upper arm, it measures the normal force applied due to muscle contraction, called muscle contraction-induced (MCI) force. FSR sensors register this applied force in terms of varying resistance. An amplifier is used to read the resistance change and output a smooth and amplified voltage signal. The output voltage signal is passed to a computer for post processing. In post processing, the voltage signal is converted to respective force measured by the FSRs. Moreover, machine learning is implemented to interpret the force signal in terms of payload. The details of all major components are described presently.

\subsection{FSR distribution}

The sensor band is comprised of an array of FSRs embedded inside a flexible strap. In the current setup, four FSR sensors of model FSR-402 are utilized, which are able to measure applied force in the range of 0.1 $10 \mathrm{~N}$.

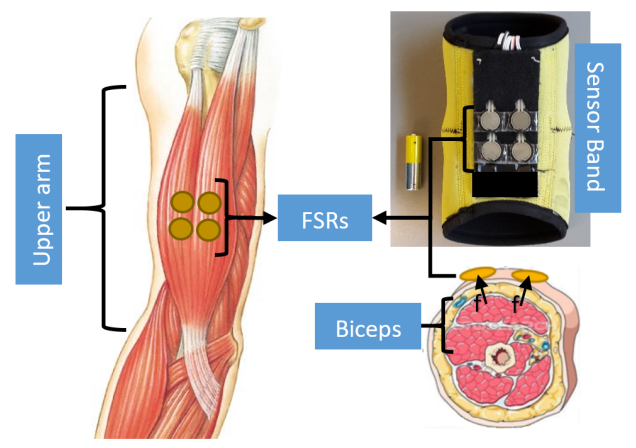

Figure 1: Sensor band working principle and it's placement on the upper arm.

FSRs distribution over the sensor band and its placement on arm are shown in Fig. 1. The sensors are distributed in a way that they can cover some areas of muscles, specially, where they can read the maximum normal force. The flexibility of strap ensures a good contact between FSRs and arm muscles, which allows the FSRs to sense the normal force exerted by the muscles on them. Moreover, the design of sensor band allows it to apply same pressure over the muscles every time the user puts it on.

\subsection{FSR-amplifier coupling}

The FSR responds to the applied force by varying its resistance. Therefore, a non-inverting amplifier is interfaced with the FSRs according to Fig. 2.

The output of the amplifier is given by the following equation,

$$
V_{\text {out }}=\left(1+\frac{R_{\text {ref }}}{R_{f s r}}\right) V_{\text {in }}
$$

where $V_{\text {out }}$ is the output voltage of the amplifier, $V_{\text {in }}$ is the input voltage to the positive terminal of the amplifier, $R_{r e f}$ is the reference resistance and $R_{f s r}$ represents the resistance of FSR. The output of the amplifier can be changed by varying $V_{i n}$ and $R_{r e f}$. In the implementation $V_{i n}$ was fixed and $R_{\text {ref }}$ was varied to read 


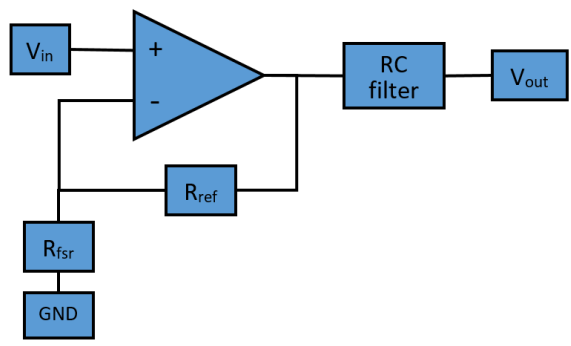

Figure 2: FSR amplification circuitry.

the desired force range of the FSR. $R_{\text {ref }}$ was finally selected, so that the full range of amplifier output is utilized and maximum resolution is obtained. In the circuit a low pass filter is also included to filter out high frequency noises.

\section{Estimation algorithm}

The algorithm of payload estimation is based on MCI force and joint orientation. In this method two MCI force profiles are modeled for carrying two different payloads across human arm range of motion in sagittal plane. The profiles are obtained using SVM based regression technique, in which MCI force is treated as output parameter and elbow and shoulder joint angles as input parameters. In the real-time testing the given MCI force is compared with the developed MCI profiles to estimate the payload.

\subsection{System setup}

The data for training SVM regression models is acquired through an instrumented passive exoskeleton. As shown in Fig. 3, the passive exoskeleton has an absolute encoder, Novotechnik RFD-4021, at elbow joint and an accelerometer ADXL 335 to monitor the upper arm movement. The output of RFD-4021 encoder determines the elbow joint angle, whereas the output of accelerometer is calibrated and mapped to shoulder joint (flexion/extension) angle as,

$$
\theta_{s}=-154.93 V_{a}^{2}+217.33 V_{a}+18.53
$$

here $V_{a}$ represents the x-axis output voltage of the accelerometer.

\subsection{Datasets}

Two datasets are recorded in the training session, namely, $\mathbf{D}_{\mathbf{1}}$ and $\mathbf{D}_{\mathbf{2}}$ to train the regression models for payload estimation. The contents of these datasets are as follows.

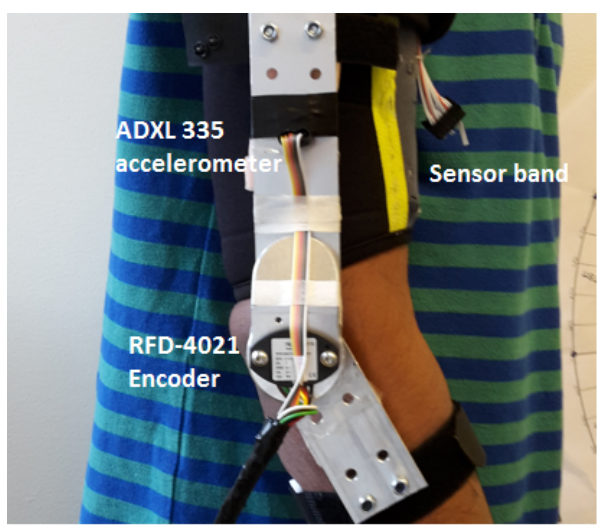

Figure 3: An instrumented passive arm exoskeleton to collect the data for SVM training.

\subsection{1 $\mathrm{D}_{1}$}

This dataset is comprised of net MCI force $f_{a}$ measured by the sensor band in carrying a payload $\rho_{a}$. In collecting the data subject lifts the payload in several elbow and shoulder joint angle configurations.

\subsection{2 $\mathrm{D}_{2}$}

This dataset is comprised of net MCI force $f_{a}$ measured by the sensor band in carrying a payload $\rho_{b}$. Arm configuration used for $\mathbf{D}_{\mathbf{1}}$ is followed in collecting this dataset.

After collecting these datasets two regression models are trained, whose details are given in forthcoming section.

\subsection{Regression models}

Both datasets, explained earlier, are used to train two regression models i.e. $\mathbf{R}_{\mathbf{1}}$ and $\mathbf{R}_{\mathbf{2}}$.

Taking $\mathbf{R}_{\mathbf{1}}$ as an example. Dataset $\mathbf{D}_{\mathbf{1}}$ is used to train this regression model with joint angles $\theta_{e}$ and $\theta_{s}$ as predictor and net MCI force $f_{a}$, sum of forces measured by FSR sensors embedded inside the sensor band, as response variable. Hence, during the real-time testing, the regression model uses the joint angles $\theta_{e}$ and $\theta_{s}$ to estimate the force, $f_{1}$, that muscle generated if a person lifts or carries payload $\rho_{a}$. Similarly, model $\mathbf{R}_{\mathbf{2}}$ is trained with dataset $\mathbf{D}_{\mathbf{2}}$. The inputs and outputs of both regression models are illustrated in Fig. 4.

\subsection{Real-time estimation model}

During real-time estimation, the algorithm first computes forces $f_{1}$ and $f_{2}$ using regression models $\mathbf{R}_{\mathbf{1}}$ and $\mathbf{R}_{2}$, respectively.

After all forces are obtained, payload is determined by following equation, 


$$
\rho=\left(\rho_{b}-\rho_{a}\right)\left(f_{a}-f_{1}\right) /\left(f_{2}-f_{1}\right)
$$

where $\rho$ represent the estimated payload.

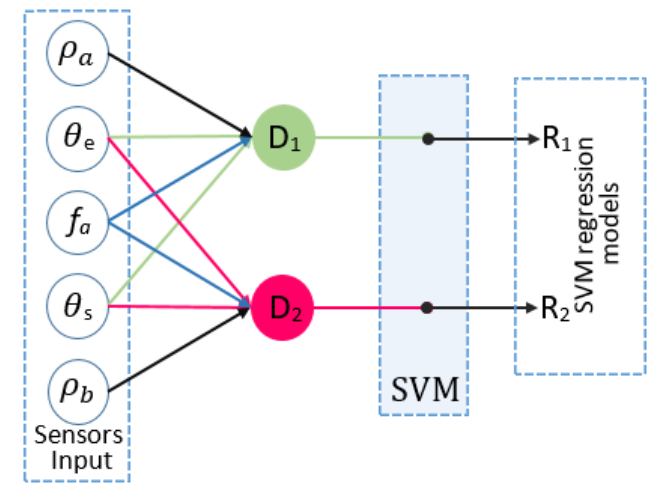

(a)

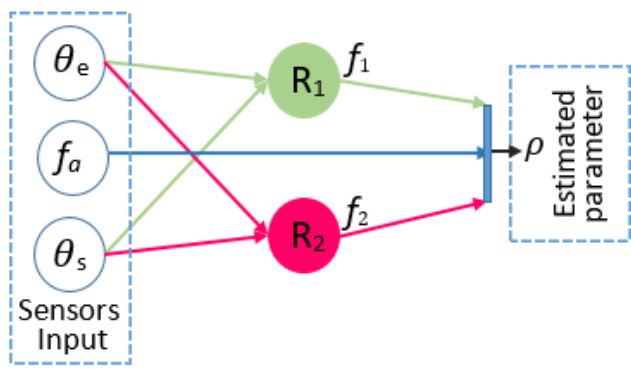

(b)

Figure 4: Flow diagram of algorithm design, (a) SVM training session to compute regression models, (b) real-time estimation of payload.

A 3D surface plot illustrating the MCI variation with respect to $\theta_{e}$ and $\theta_{s}$ is shown in Fig. 5.

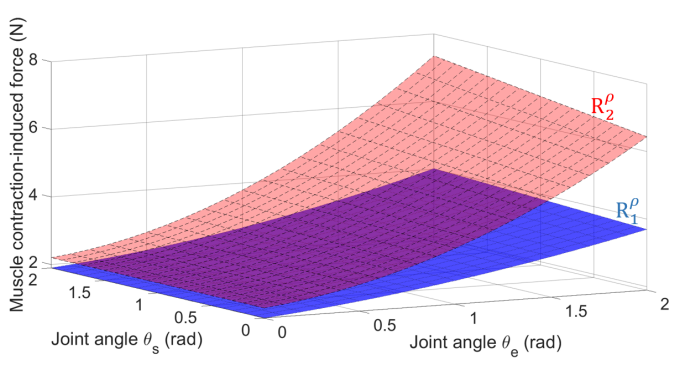

Figure 5: Illustration of payload estimation when two payloads are used for training the regression models.

\section{Payload estimation}

Experiments were carried out to test the performance of the developed payload determination method. The experiments of payload estimation first include training of SVM regression models, which is followed by realtime testing of the developed estimation method.

\subsection{Data collection protocol}

A MATLAB based GUI is developed to collect the data, which is comprised of MCI force readings from sensor band, elbow joint and shoulder joint angles. The GUI allows the data to be collected at a frequency of $200 \mathrm{~Hz}$ and sorting out the necessary information for training session.

The protocol of data collection involves a set of static postures, shown in Fig. 6, that the user maintains for a few seconds. The detailed description of protocol is as follow.

- Subject wears the sensor band and passive exoskeleton as shown in Fig. 3.

- Subject is free of payload, i.e. $\rho_{a}=0 \mathrm{~kg}$, and keeps the elbow and shoulder angles according to Fig. 6(a) for $10 \mathrm{~s}$.

- Subject rests for $20 \mathrm{~s}$ and raises his/her arm to the next configuration as shown in Fig. 6(b) and maintains the pose for $10 \mathrm{~s}$.

- Subject repeats the task for all the positions shown in Fig. 6, with a rest for $20 \mathrm{~s}$ in between each position.

- After completing all positions subject rests for 10 minutes and repeats the whole process for payload $\rho_{b}=2.5 \mathrm{~kg}$.

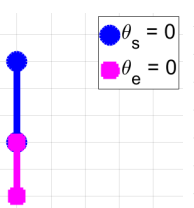

(a)

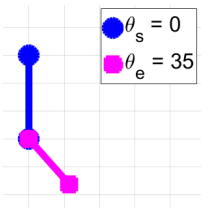

(b)

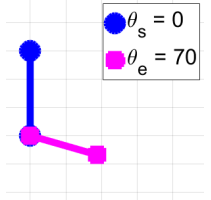

(c)

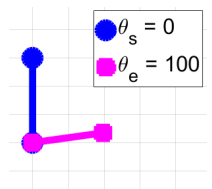

(d)

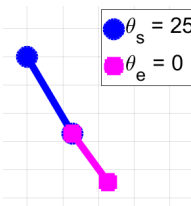

(e)

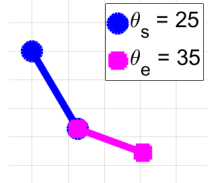

(f)

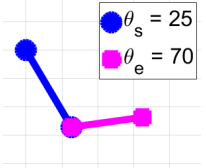

(g)

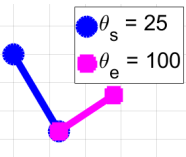

(h)
Figure 6: Joint configurations selected for collecting sensors data.

The data collected in these experiments is divided into two sets i.e. $\mathbf{D}_{\mathbf{1}}$ and $\mathbf{D}_{\mathbf{2}}$, which is followed by the training of regression models $\mathbf{R}_{\mathbf{1}}$ and $\mathbf{R}_{\mathbf{2}}$, respectively. 


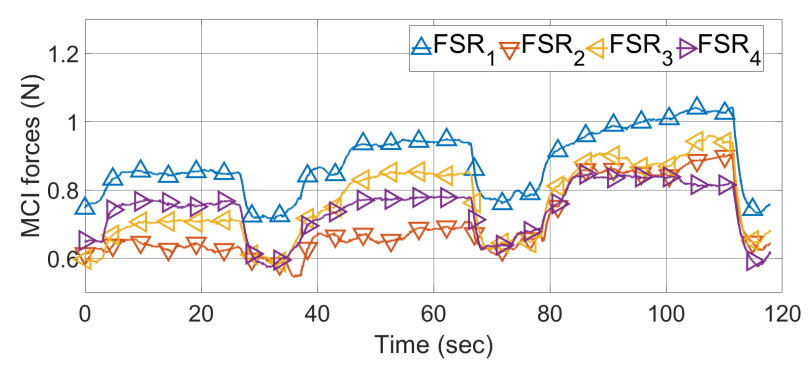

(a)

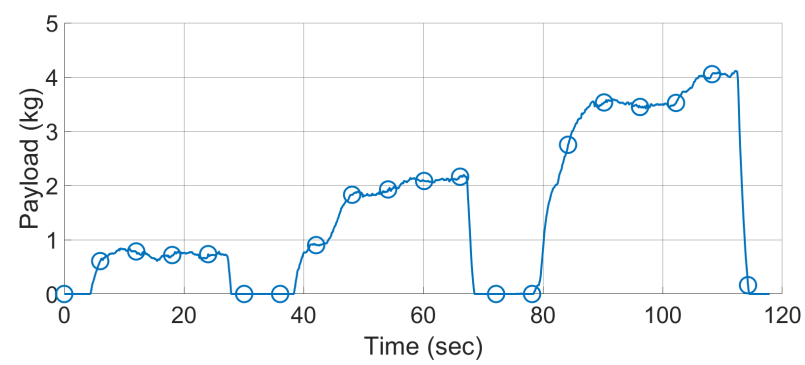

(b)

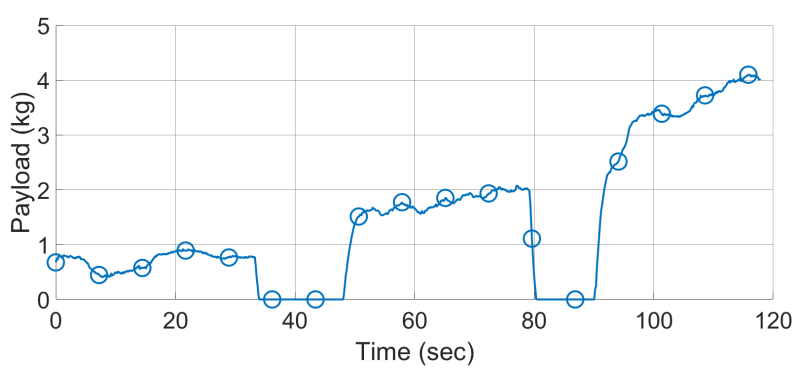

(c)

Figure 7: Payload estimation testing results, (a) MCI forces measured by sensor band for a configuration of $\theta_{e}$ $=76^{\circ}$ and $\theta_{s}=34^{\circ}$, (b) payload estimated for case (a), and (c) payload estimated for a configuration of $\theta_{e}=76^{\circ}$ and $\theta_{s}=52^{\circ}$.

\subsection{Results of real-time testing}

Five healthy subjects, aged between 20-30, participated in this experiment. Subjects were provided with written consent forms prior to the experiments. Moreover, experiments were performed with ethical approval obtained from ethical committee, Region Nordjylland, Denmark.

In this testing the regression models for payload estimation were trained and tested for each subject separately. After training the regression models, real-time testing was carried out with the passive exoskeleton, in which three different payloads in the range of $0 \mathrm{~kg}$ to $5 \mathrm{~kg}$ were lifted by the subjects.

Figure 7 shows the test results with three payloads $\rho_{1}=0.8 \mathrm{~kg}, \rho_{2}=2.5 \mathrm{~kg}$ and $\rho_{3}=4 \mathrm{~kg}$, which were held by Subject 1 at his hand sequentially.

The testing is static, which means that the elbow and shoulder joint angles remain fixed. Figure 7(a) displayed raw data of FSR readings measured for angles $\theta_{e}=76^{\circ}$ and $\theta_{s}=34^{\circ}$. Figure $7(\mathrm{~b})$ shows the estimated payload. Another result of payload estimation is shown in Fig. 7(c), with the arm configuration slightly changed i.e. $\theta_{e}=76^{\circ}$ and $\theta_{s}=52^{\circ}$.

The testing shows that the developed method can determine the payload level correctly and close to real values for varying configurations. It is also noticed in Figs. 7(b) and 7(c) that even though the SVM models were trained for up to $2.5 \mathrm{~kg}$ payload, the sensor can even estimate correctly the payload level higher than the top level used in the training.

The results of all the other subjects are shown in Fig. 8, displaying errors in payload estimation. The error in estimated payload is computed for all the tasks performed in saggital plane, as the training condition in payload estimation included arm configurations for different shoulder and elbow joint angles.

Figures 8(a) and 8(b) show absolute and relative error of estimation, respectively. Absolute error is computed as the difference between actual and estimated payload value, whereas, relative error is obtained by normalizing the error w.r.t the maximum payload. It can be seen in Fig. 8(a) that the mean value of absolute error varies from $0.14 \mathrm{~kg}$ to $0.37 \mathrm{~kg}$. In Fig. 8(b) mean value of relative error varies from 0.07 to 0.17 for the five subjects.

\section{Exoskeleton control}

With the developed sensor for payload determination, we conducted further experiments of physical assistance control on a 4-DOF upper-body exoskeleton (see Fig. 9), developed at AAU, Aalborg, Denmark Bai et al. (2017). The exoskeleton has 3 ac- 


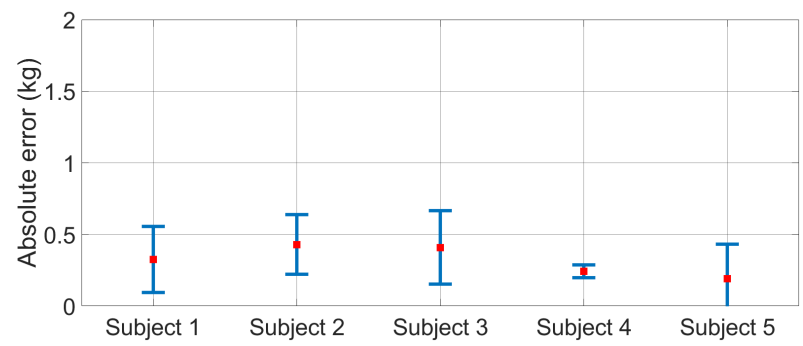

(a)

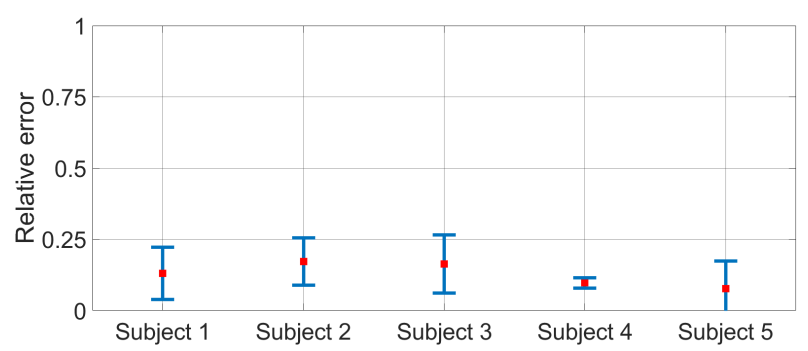

(b)

Figure 8: Errors of estimation, (a) absolute error, and (b) relative error, where red dot refers to the mean values.

tive joints (i.e. elbow flexion/extension, shoulder flexion/extension and shoulder abduction/adduction) and 1 passive joint (shoulder internal/external rotation). In these experiments shoulder abduction/adduction and internal/external rotation motions are restricted and only elbow/shoulder flexion/extension motors are actuated.

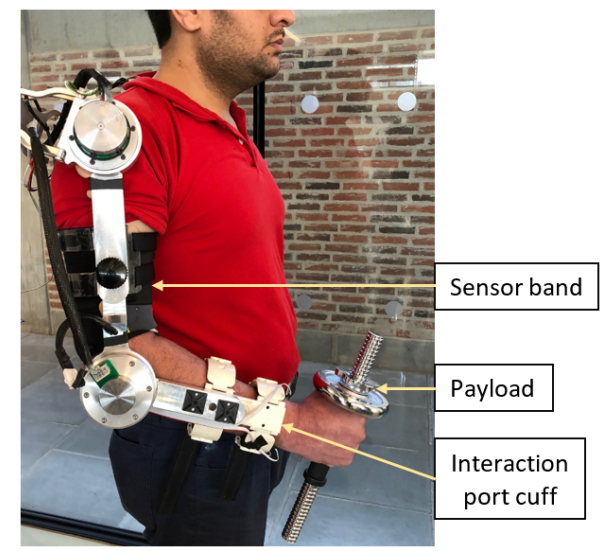

Figure 9: Upper-body exoskeleton for physical assistance testing.

The exoskeleton adapts admittance control and gravity compensation. Admittance control is implemented to control the elbow actuator motion, whereas, grav- ity compensation is implemented to provide support at shoulder joint. The block diagram of the control algorithm is presented in Fig. 10.

The control input $u$ relayed to exoskeleton is,

$$
u=\left[\begin{array}{ll}
\tau_{e} & \tau_{s}
\end{array}\right]
$$

where $\tau_{e}$ represents the elbow actuator control input and $\tau_{s}$ the shoulder actuator control input.

The elbow torque is dependent on interaction torque $\tau_{\text {int }}$ at attachment cuff and assistance torque $\tau_{a}$ associated to the payload $\rho$, which are computed by

$$
\begin{gathered}
\tau_{\text {int }}=f_{p} \cdot r \\
\tau_{a}=k_{A L} \rho g l_{p} \sin \left(\theta_{e}+\theta_{s}\right)
\end{gathered}
$$

where $f_{p}$ is the interaction port force that is measured from the port cuff in Fig. 9, $r$ is the distance from the middle point of the interaction port cuff to elbow joint, $g$ is the gravity acceleration, $l_{p}$ is the distance from elbow joint to the center of palm and $k_{A L}$ is assistance coefficient, which is computed through

$$
k_{A L}=\frac{A}{1-A}
$$

where $A \in[0,1)$, defines the percentage of assistance provided by the exoskeleton. Eq. 7 ensures the equilibrium between human effort and exoskeleton's assistance for a desired value of $A$, in order to perform the task jointly.

After determining the interaction and assistive force, the corresponding joint torque is computed, which is followed by the admittance filter $(\mathrm{Y}(\mathrm{s}))$ to obtain the desired velocity. Both are mathematically represented as,

$$
\begin{gathered}
\tau_{\text {net }}=\tau_{\text {int }}+\tau_{a} \\
Y(s)=\frac{\omega_{d}(s)}{\tau_{\text {net }}(s)}=\frac{1}{B s+D+\frac{K}{s}}
\end{gathered}
$$

where $\tau_{\text {net }}$ is the required joint torque, $\omega_{d}$ is the desired velocity and $B, D$ and $K$ represent the inertia, damping and stiffness parameters of the admittance filter. Furthermore, the desired joint velocity is tracked through a PI controller which outputs the control input $\tau_{e}$ given by,

$$
\tau_{e}=k_{p} \omega_{e}+k_{i} \int \omega_{e} d t
$$

where $k_{p}$ and $k_{i}$ are the proportional and integral gains, respectively. $\omega_{e}$ is the error signal and is given by,

$$
\omega_{e}=\omega_{d}-\omega_{a}
$$




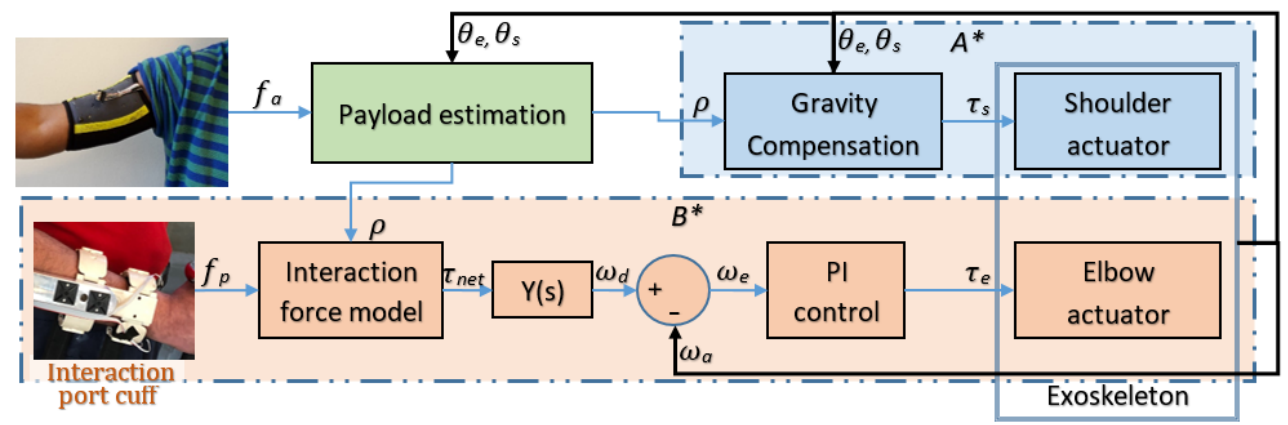

Figure 10: Exoskeleton control block diagram, where $\mathrm{A}^{*}$ and $\mathrm{B}^{*}$ are two blocks for control of shoulder and elbow joint, respectively.

Table 1: Exoskeleton and control parameters

\begin{tabular}{cccc}
\hline \hline Parameter & Value & Parameter & Value \\
\hline$m_{e}$ & $1.39 \mathrm{~kg}$ & $A$ & 0.5 \\
$m_{s}$ & $0.307 \mathrm{~kg}$ & $k_{s}$ & 50 \\
$l_{e}$ & $0.165 \mathrm{~m}$ & $B$ & $0.05 \mathrm{kgm}^{2}$ \\
$l_{s}$ & $0.15 \mathrm{~m}$ & $D$ & $0.105 \mathrm{Nsm}^{-1}$ \\
$l_{s e}$ & $0.33 \mathrm{~m}$ & $K$ & $0 \mathrm{Nm}^{-1}$ \\
$r$ & $0.27 \mathrm{~m}$ & $k_{p}$ & 1 \\
$r_{f r}$ & $0.30 \mathrm{~m}$ & $k_{i}$ & 0.0067 \\
\hline \hline
\end{tabular}

where $\omega_{a}$ represents the actual joint angular velocity.

The shoulder actuation is controlled with gravity compensation. The shoulder joint control input $\tau_{s}$ is computed by,

$\tau_{s}=m_{s} g l_{s} \sin \theta_{s}+\left(m_{e}+\rho k_{A L}\right) g\left(l_{e} \sin \left(\theta_{e}+\theta_{s}\right)+l_{s e} \sin \theta_{s}\right)$

where $m_{e}$ and $m_{s}$ represent the forearm and upper arm link mass, $l_{e}$ is the distance from elbow joint to the center of mass of forearm link, $l_{s}$ is the distance from shoulder joint to the center of mass of upper arm link and $l_{s e}$ is the distance from shoulder joint to elbow joint. The values of the exoskeleton and control parameters are provided in Table 1.

The results of the estimated payload and physical assistance provided by exoskeleton are shown in Fig. 11. Figure 11(a) shows the results of subject holding a payload of $2.5 \mathrm{~kg}$ without wearing the active exoskeleton, in an arm configuration i.e. $\theta_{e}=88^{\circ}$ and $\theta_{s}=$ $24^{\circ}$ approximately. It can be seen that the estimated payload value is close to the actual payload value i.e. $2.5 \mathrm{~kg}$.

Figures 11(b) and 11(c) show the result of payload estimation and joint torques, respectively, for subject carrying the same payload while wearing the exoskeleton, in arm configuration similar to the payload carrying task without exoskeleton. In this test, the exoskele-

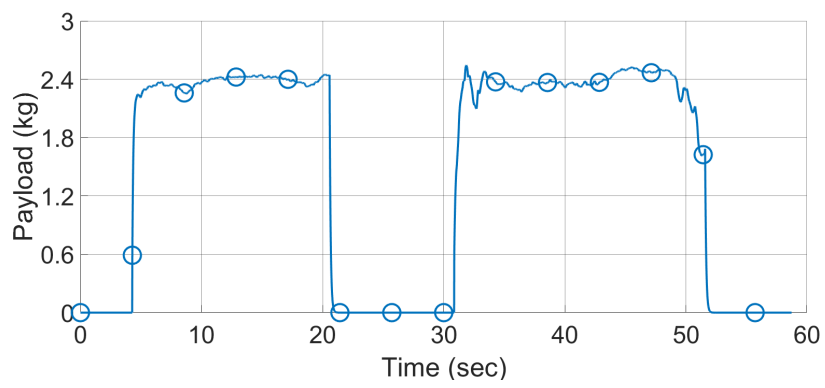

(a)

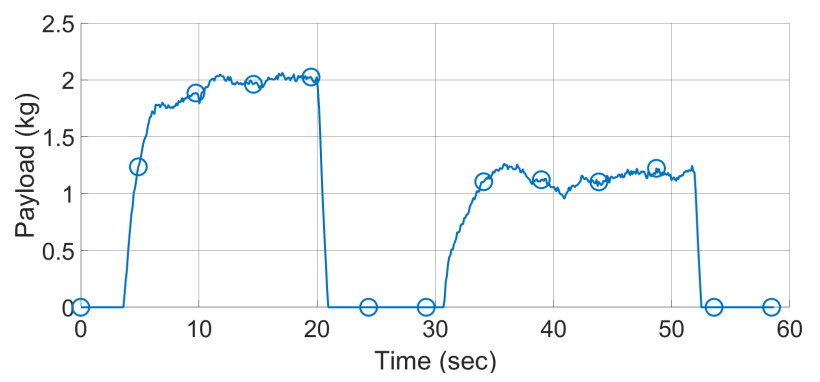

(b)

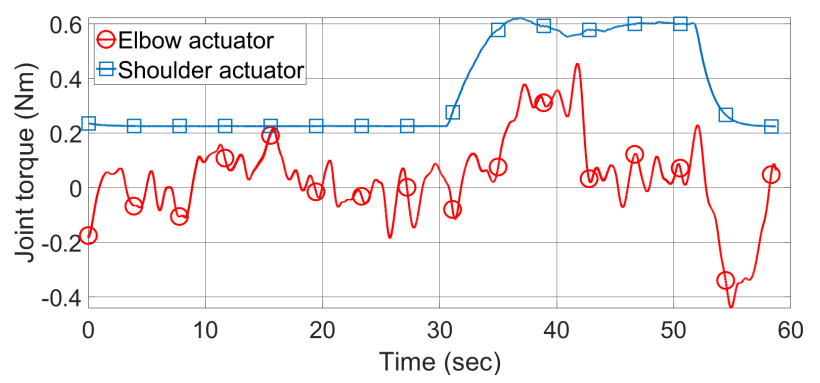

(c)

Figure 11: Exoskeleton control results, (a) estimated payload without exoskeleton, (b) estimated payload with exoskeleton and (c) torques provided by exoskeleton at each joint, first in transparent mode (3-21 s) and then in assistive mode (31-52 s). 
ton worked in transparent mode for $\mathrm{t}=3-21 \mathrm{~s}$ and then in assistive mode for $\mathrm{t}=31-52 \mathrm{~s}$.

It can be seen that in transparent mode the estimated payload is approximately $2 \mathrm{~kg}$, which is slightly less than the estimated payload without exoskeleton that was nearly $2.4 \mathrm{~kg}$. This can be justified as in transparent mode the exoskeleton provides slight assistance and therefore, effort exerted by upper arm muscles will decrease. The decrease in muscle effort results in decrease of MCI force. Since our method of payload estimation is based on upper arm MCI force, therefore with the decease in MCI force the algorithm will estimate reduced payload.

Comparing the results of payload carrying task without exoskeleton and payload carrying task with exoskeleton in assistive mode, it can be seen that the estimated payload value decreased from $2.4 \mathrm{~kg}$ to 1.2 $\mathrm{kg}$, respectively. The decrease in payload value justifies that the exoskeleton is providing assistance in load carrying task. As explained earlier, the payload estimation algorithm is based on MCI force of upper arm muscles. With assistance from exoskeleton, the upper arm muscles activity will decrease. The decrease in muscle activity causes the MCI force to decrease. Therefore, it is seen that in assistive exoskeleton control the $2.5 \mathrm{~kg}$ payload is estimated, or in other words, felt as $1.2 \mathrm{~kg}$ payload carried by the subject.

\section{Discussion}

In this work, we developed a novel method for the estimation of payload. The developed method shows some advantages for exoskeleton assistance control.

The new method is advantageous in reducing complexity in upper arm exoskeleton control. Conventionally, in order to provide assistance at elbow and shoulder joint, activities of muscles that govern the elbow and shoulder joint movement need to be observed. This makes the system physically and computationally complex. In this work only biceps muscle readings along with elbow and shoulder joint encoder are used to estimate the payload. The method has significantly reduced the complexity not only in real-time operations but also in training sessions. Additionally, the sensor band can be readily and conveniently put on human arms and used when needed. Moreover, unlike EMG based solutions FSR output is not affected by the skin condition and slight displacement from optimum position does not affect the sensor output.

It is noticed that the developed method has some limitations. One limitation is that the sensor band readings can be affected by external interference. Sensor band primarily measures the contact force that can result from external contact, which can be cuffs to hold the exoskeleton. Therefore, the design of the cuffs needs to be in a way to not come in contact with the FSRs. Possible saturation of FSR sensor output is another limitation. The amplifier is thus required to be properly tuned in order to avoid saturation. These limitations need further improvements in future research.

\section{Conclusion}

The paper presents a novel method of estimating payload using an FSR based sensor band. The sensor band is able to measure the muscle contraction-induced forces. Machine learning is used to process and interpret the readings of the sensor, which yields the payload estimated. The method has been tested and validated in a series of testing and then applied to the assistive control of an upper-body exoskeleton to provide required assistance.

The main contribution of the presented work lies in the convenient and accurate estimation of payload using an FSR based sensor band, which makes it simple and effective for practical use. In this work, the accuracy of payload determination is tested through experiments. This method also makes it possible for exoskeletons to control physical assistance with a simple setup such as the FSR based sensor band. The experimental work on the exoskeleton control for load carrying assistance justifies this possibility, which is another contribution of this work.

In the presented work sensor band is applied for upper-body exoskeleton control. The sensor technology can also be used in other systems, for example, soft/rigid hand exoskeleton or lower limb exoskeleton to detect the load level. The future work will focus on more comprehensive testing of the payload estimation and extending the current work to full upper-body exoskeleton control. Moreover, comparative study of different regression algorithms for the estimation of payload will be considered. The developed sensor technology with various control strategies will also be considered.

\section{Acknowledgment}

The authors would like to thank EU AAL Programme's funding bodies to support the project AXO-SUIT (http://www.axo-suit.eu/) and Innovation Fund Denmark's support to EXO-AIDER (https://www.exoaider.dk/). 


\section{References}

Abdallah, I. B., Bouteraa, Y., and Rekik, C. Design and development of $3 \mathrm{D}$ printed myoelectric robotic exoskeleton for hand rehabilitation. International Journal on Smart Sensing 6 Intelligent Systems, 2017. 10(2). doi:10.21307/ijssis-2017-215.

Bai, S., Christensen, S., and Islam, M. R. U. An upper-body exoskeleton with a novel shoulder mechanism for assistive applications. In 2017 IEEE International Conference on Advanced Intelligent Mechatronics (AIM). pages 1041-1046, 2017. doi:10.1109/AIM.2017.8014156.

Bai, S., Virk, G. S., and Sugar, T. Wearable Exoskeleton Systems: Design, Control and Applications. Institution of Engineering and Technology, 2018. doi:10.1049/PBCE108E.

Castro, M. N., Rasmussen, J., Andersen, M. S., and Bai, S. A compact 3-DOF shoulder mechanism constructed with scissors linkages for exoskeleton applications. Mechanism and Machine Theory, 2019. 132:264-278. doi:10.1016/j.mechmachtheory.2018.11.007.

Cho, E., Chen, R., Merhi, L.-K., Xiao, Z., Pousett, B., and Menon, C. Force myography to control robotic upper extremity prostheses: a feasibility study. Frontiers in Bioengineering and Biotechnology, 2016. 4:18. doi:10.3389/fbioe.2016.00018.

Christensen, S. and Bai, S. Kinematic analysis and design of a novel shoulder exoskeleton using a double parallelogram linkage. Journal of Mechanisms and Robotics, 2018. 10(4):041008. doi:10.1115/1.4040132.

Cui, X., Chen, W., Jin, X., and Agrawal, S. K. Design of a 7-DOF cable-driven arm exoskeleton (CAREX-7) and a controller for dexterous motion training or assistance. IEEE/ASME Transactions on Mechatronics, 2016. 22(1):161-172. doi:10.1109/TMECH.2016.2618888.

Fan, Y. and Yin, Y. Active and progressive exoskeleton rehabilitation using multisource information fusion from EMG and force-position epp. IEEE Transactions on Biomedical Engineering, 2013. 60(12):33143321. doi:10.1109/TBME.2013.2267741.

Gunasekara, J., Gopura, R., Jayawardane, T., and Lalitharathne, S. Control methodologies for upper limb exoskeleton robots. In 2012 IEEE/SICE International Symposium on System Integration (SII). pages 19-24, 2012. doi:10.1109/SII.2012.6427387.
Hsieh, H.-C., Chen, D.-F., Chien, L., and Lan, C.-C. Design of a parallel actuated exoskeleton for adaptive and safe robotic shoulder rehabilitation. IEEE/ASME Transactions on Mechatronics, $2017 . \quad 22(5): 2034-2045$. doi:10.1109/TMECH.2017.2717874.

Huang, J., Huo, W., Xu, W., Mohammed, S., and Amirat, Y. Control of upper-limb power-assist exoskeleton using a human-robot interface based on motion intention recognition. IEEE Transactions on Automation Science and Engineering, 2015. 12(4):1257-1270. doi:10.1109/TASE.2015.2466634.

Islam, M. R., Xu, K., and Bai, S. Position sensing and control with FMG sensors for exoskeleton physical assistance. In International Symposium on Wearable Robotics. pages 3-7, 2018. doi:10.1007/978-3-03001887-0_1.

Islam, M. R. U. and Bai, S. Intention detection for dexterous human arm motion with FSR sensor bands. In Proceedings of the Companion of the 2017 ACM/IEEE International Conference on Human-Robot Interaction. pages 139-140, 2017. doi:10.1145/3029798.3038377.

Jiang, X., Chu, H. T., Xiao, Z. G., Merhi, L.-K., and Menon, C. Ankle positions classification using force myography: An exploratory investigation. In 2016 IEEE Healthcare Innovation Point-Of-Care Technologies Conference (HI-POCT). pages 29-32, 2016. doi:10.1109/HIC.2016.7797689.

Kadkhodayan, A., Jiang, X., and Menon, C. Continuous prediction of finger movements using force myography. Journal of Medical and Biological Engineering, 2016. 36(4):594-604. doi:10.1007/s40846-0160151-y.

Keller, U., van Hedel, H. J., Klamroth-Marganska, V., and Riener, R. ChARMin:The first actuated exoskeleton robot for pediatric arm rehabilitation. IEEE/ASME Transactions on Mechatronics, $2016 . \quad 21(5): 2201-2213$. doi:10.1109/TMECH.2016.2559799.

Kiguchi, K. and Hayashi, Y. An EMG-based control for an upper-limb power-assist exoskeleton robot. IEEE Transactions on Systems, Man, and Cybernetics, 2012. 42(4):1064-1071. doi:10.1109/TSMCB.2012.2185843.

Lee, H.-D., Lee, B.-K., Kim, W.-S., Han, J.-S., Shin, K.-S., and Han, C.-S. Human-robot cooperation control based on a dynamic model of 
an upper limb exoskeleton for human power amplification. Mechatronics, 2014. 24(2):168-176. doi:10.1016/j.mechatronics.2014.01.007.

Leonardis, D., Barsotti, M., Loconsole, C., Solazzi, M., Troncossi, M., Mazzotti, C., Castelli, V. P., Procopio, C., Lamola, G., Chisari, C., et al. An EMGcontrolled robotic hand exoskeleton for bilateral rehabilitation. IEEE Transactions on Haptics, 2015. 8(2):140-151. doi:10.1109/ТОH.2015.2417570.

Li, Z., Wang, B., Sun, F., Yang, C., Xie, Q., and Zhang, W. sEMG-based joint force control for an upper-limb power-assist exoskeleton robot. IEEE Journal of Biomedical and Health Informatics, 2013. 18(3):1043-1050. doi:10.1109/JBHI.2013.2286455.

Mangukiya, Y., Purohit, B., and George, K. Electromyography EMG sensor controlled assistive orthotic robotic arm for forearm movement. In 2017 IEEE Sensors Applications Symposium (SAS). pages 1-4, 2017. doi:10.1109/SAS.2017.7894065.

McDonald, C. G., Dennis, T. A., and O'Malley, M. K. Characterization of surface electromyography patterns of healthy and incomplete spinal cord injury subjects interacting with an upper-extremity exoskeleton. In 2017 International Conference on Rehabilitation Robotics (ICORR). pages 164-169, 2017. doi:10.1109/ICORR.2017.8009240.

Mghames, S., Laghi, M., Della Santina, C., Garabini, M., Catalano, M., Grioli, G., and Bicchi, A. Design, control and validation of the variable stiffness exoskeleton FLEXO. In 2017 International Conference on Rehabilitation Robotics (ICORR). pages 539-546, 2017. doi:10.1109/ICORR.2017.8009304.

Rahman, M. H., Ochoa-Luna, C., Saad, M., and Archambault, P. EMG based control of a robotic ex- oskeleton for shoulder and elbow motion assist. Journal of Automation and Control Engineering, 2015. 3(4). doi:10.12720/joace.3.4.270-276.

Rosen, J., Brand, M., Fuchs, M. B., and Arcan, M. A myosignal-based powered exoskeleton system. IEEE Transactions on Systems, Man, and Cyberneticspart A: Systems and humans, 2001. 31(3):210-222. doi:10.1109/3468.925661.

Sadarangani, G. P. and Menon, C. A preliminary investigation on the utility of temporal features of force myography in the two-class problem of grasp vs. no-grasp in the presence of upper-extremity movements. Biomedical Engineering online, 2017. 16(1):59. doi:10.1186/s12938-017-0349-4.

Tang, Z., Zhang, K., Sun, S., Gao, Z., Zhang, L., and Yang, Z. An upper-limb power-assist exoskeleton using proportional myoelectric control. Sensors, 2014. 14(4):6677-6694. doi:10.3390/s140406677.

Xiao, Z. G., Elnady, A. M., and Menon, C. Control an exoskeleton for forearm rotation using FMG. In 5th IEEE RAS/EMBS International Conference on Biomedical Robotics and Biomechatronics. pages 591-596, 2014. doi:10.1109/BIOROB.2014.6913842.

Xiao, Z. G. and Menon, C. Counting grasping action using force myography: an exploratory study with healthy individuals. JMIR Rehabilitation and Assistive technologies, 2017. 4(1):e5. doi:10.2196/rehab.6901.

Zhou, L., Bai, S., Andersen, M. S., and Rasmussen, J. Modeling and design of a spring-loaded, cabledriven, wearable exoskeleton for the upper extremity. Modeling, Identification and Control, 2015. 36(3):167-177. doi:10.4173/mic.2015.3.4. 